\title{
EXPERIENCES IN PHOTOGRAMMETRIC AND LASER SCANNER SURVEING OF ARCHITECTURAL HERITAGE
}

\author{
Mauro CAPRIOLI ${ }^{1}$, Maurizio MINCHILLI², Alfredo SCOGNAMIGLIO ${ }^{1}$ \\ ${ }^{1}$ Department of "Vie e Trasporti", Polytechnic of Bari, \\ V. Orabona, 470125 BARI Italy \\ m.caprioli@poliba.it \\ ${ }^{2}$ Department of Architecture, Design and Urbanism, University of Sassari, \\ minchilli@uniss.it
}

Keywords : photogrammetry, laser scanning

\begin{abstract}
The integration of different methodologies and simplification of procedures is surely one of the most important current themes in survey techniques for researching cultural heritage, especially for architectural heritage. The traditional methods for the metric survey of an architectural building (including micro geodesy and the stereophotogrammetric survey) are currently being consolidated. However, in the field of the architectural, artistic and archaeological survey, new technologies are now available and this knowledge needs to be more widely used, especially where the heritage is greater and so maintenance is as necessary as it is onerous. This report proposes some operative considerations, which derive from experience developed using the most modern technologies available, in order to compare and discuss the achievable accuracies (even if they are not always strictly necessary) and the operating modalities, especially for architectural surveys. So, the aim of this report is to verify that the simplification of procedures and cheaper solutions do not bring variations to the necessary metric precision or expert photointerpretation analysis. However, it is clear that, without considering the purposes and objectives of the survey, it would not be correct to appraise the survey methods and to classify those using reliability and accuracy criteria. In fact, the objective of a survey determines which tool will be used, according to the requests of the survey client to the survey expert.
\end{abstract}

\section{DIGITAL PHOTOGRAMMETRY}

Since the 1980s terrestrial photogrammetry methodology has been meaningfully changed in terms of methodology, performance and operation. These years have seen first the passage from analogical resolution tools (which were very widely used in Italy at that time) to analytical tools and then the passage to hybrid trials (analogical resumptions digital restitution) and finally to entirely digital technologies. New technologies and techniques were gradually developed for data acquisition (photo grammatical scanner and metric digital cameras), for the elaboration of numerical data (homologous image correlation), for representation (2D and 3D Cad, raster/vector file hybrid treatment, simulation, animation and virtual reality) and for the treatment of information and spatial analyses (georeferential Informative Systems in the architectural and archaeological fields). The improvement in survey methods for monuments and historical places have provided an important contribution to describing and monitoring Cultural Heritage, for the maintenance and restoration of monuments, objects and architectural places as well as helping historical, architectural and archaeological research. However, photogrammetry is still used to obtain geometric information such as position, measurements and the shape of objects captured by photographic images, without measuring and analyzing them directly. Currently, the optical system (for coordinate restitution of a point in three-dimensional space by the minimal intersection between two optic rays that connect a point on the object with the center of the camera objective and with the image point) guarantees great freedom of action, just working directly on digital images. Furthermore, the presence of more than 3 projected rays of this kind (if objects appear on three or more photos) makes it possible to seek a general solution for the identification of survey point coordinates (Bundle solution) by using all available measurements together (photographic and other). When the object is on a plane it is very common to use single-frame shots because of the quick elaboration for high-resolution and large dimension images. In addition, digital photo-adaptation techniques, expressed as geometric transformations of images and projections on spatial models are frequently used. This is the case when the surface of an object in space (digital surface model DSM) is already known, so it is important to know details of the representation (weaving, models, lesions, material analysis etc.). Knowledge of the camera orientation parameters greatly helps the geometric construction of a reliable model and, in recent years, laboratory autosetting-calibration methodologies have been made available to the final user. In any case, external-orientation reconstruction requires 3D 
point measurements, obtained by traditional techniques. When the measurements of an object or its geometry are not required, at least two photographic images of it can be useful in order to exploit the stereoscopic-vision characteristics given by two images of the same object, observed simultaneously in an independent way with two eyes.

The classical-photogrammetrical restitution products are vectorial drawings of an object and, according to classical architectural drawing conventions, this describes plans, prospectuses and sections, but also among others threedimensional wireframe models, surfaces models (DSM), coordinated points lists. Photogrammetrical restitution instruments can be equipped with attributes in order to obtain more rapidly updated information about the object, for example material, state of damage or any other characteristic that is useful in order to amplify the knowledge of the architectural object. Building-Volume Informative Systems need three-dimensional GIS software. These developing procedures should allow topological and spatial analyses underlining mutual interactions between objects in threedimensional space.

\section{1 "Bundle restitution"}

In many cases using stereo-images alone is not enough for the reconstruction of objects with complex forms such as building volume, therefore a larger number of photos are used to totally reconstruct such objects. In these cases, the simultaneous resolution of the orientation of all the photos is recommended to obtain homogeneous solutions (bundle block adjustment). The terrestrial applications and development of the star-triangulation-projection software began in the 1980s with BINGO industrial applications. This kind of software is commercially available and has simple interfaces. Effecting a photographic camera calibration for each single survey (on-the-job) is another advantage because this method helps to increase the survey accuracy and is used with images taken using non-metric photographic cameras. In the case of multiple captures and in the case of surveys with multiple support-points, the photogrammetrical survey technique is more accessible and flexible because it uses uncalibrated photographic cameras. The photographs can be realized with different capture-angles, without using parallel sights (normal case) and from pre-set distances, as used in classic photogrammetry. The possibility to use horizontally and vertically convergent oblique photos, and using different photographic cameras and different lenses, allows a large choice of photographic shooting points. The photographic acquisition method has to satisfy the necessity that each point is intersected by at least two optical rays with a satisfactory angle, according to the desired metric accuracy.

In this method it is possible to use additional knowledge such as parallelism, surface flatness and angle orthogonality in order to reconstruct a more correct three-dimensional geometric model of the object. All the parameter measurements and calculations are realized using the ordinary least squares statistical compensation method and, thanks to system redundancy, it is possible to locate coarse errors and to improve the accuracy and reliability of results. This method gives very appreciable and accurate results, useful in Cad software in which it is possible to manipulate the obtained geometric data. As already explained, the results obtained by this procedure are usually 3D wireframe models or digital surfaces models of the object (DSM) or they can also be entities and points that are classified by assigned their attributes. The graphic products are 3D digital models and they can also be completed with the surface "texture", obtained directly from the photographs.

\subsection{Image acquisition Systems}

Before the availability of totally digital processes, using expensive and very specialized equipment (such as photographic metric cameras) limited the applications of architectural photogrammetry, because it was necessary to know the inside orientation parameters of the cameras. These instruments were expensive and so not widely used because it was necessary to use large format cameras (in order to read the images in a better way) and lenses were built and calibrated to have very small radial distortion. The important development and commercial availability of digital image acquisition systems has allowed lower prices and a widened market. The main advantage of these systems is the possibility to directly acquire digital images, that can then be processed in a numerical programming environment, without passing through film development, the possible photograph or acquisition by a photogrammetrical scanner (because they all introduced big or small geometric survey alterations). The digital process already allows greater productivity, related to the intrinsic automation possibilities of electronic computation and it also allows the contemporary use of vector-data and "raster" data in a single working session. Because digital aerial photogrammetric cameras are cheaper, the global digitization process has been quite fast in architectural photogrammetry: sometimes specialized digital metric cameras are produced and high resolution semi-professional cameras are used more. In summary the advantages are:

- Unique data-flow with the possibility of on-line workmanship

- Considerable automation possibilities

- Good geometric characteristics

- Independence from the film development process

- quality control of the acquired images

- Inexpensiveness of the system Components 
Which kind of photographic camera it is better to use is not an easy question to answer because different factors can induce one choice rather than another one. Often, a photogrammetrical survey can be complex (sometimes the subject of the survey itself is complex), the available budget can vary, the time available for operations can be restricted because of external factors such as emergencies caused by catastrophic events.

Thus, these factors influence the choice of the system to use and also adding variables such as the need for color, scale representation and necessary resolution, it is clear that the previous question has many possible answers. In conclusion, it is important to know all the factors and survey "objectives" in order to choose the best acquisition system. Some important considerations, derived from classical methodological experience, are as valid as actual technologies:

- Using large format sensors, according to economic availability;

- Using fixed focal lenses for better resolution and more distortion containment;

- Using lenses with manual focus setting, that are pre-set on calibration;

- Choosing a metal support structure in order to have better mechanical stability than using auto-calibration orientations;

- Using a camera stand and a low sensitivity sensor (less noise in the digital image)

\subsection{Systems and methods for architectural photogrammetry}

Most of commercial digital photogrammetry workstations (DPW) are set out primarily for stereoscopic image restitution, aerial triangulation, extraction of Digital Terrain Models (DTM) and production of ortho-photographs from aerial-terrestrial stereo images. In fact, cheaper systems are sufficient to reconstruct a three-dimensional model of the architectural object, but they do not assure metric precision accuracy, in which acquisition and elaboration tools have to be of high quality. These systems can be used by photogrammetry experts and also by other professional figures who are interested in documenting maintaining and restoring. Architectural Heritage. Software improves day by day and three-dimensional object models are easier and more accurate to realize. Examples of current software and systems are introduced here. To compare different systems, it is important to focus on the following problems: ease of use of the system, data flow, project management, data importation and exportation (image formats, internal and external orientation parameters, etc.), internal orientation, external orientation (in one or two steps only), object reconstruction, consistent, accurate and reliable topological results, the necessary photogrammetrical knowledge to manage the system.

\subsection{Monoscopic systems of restitution and multiple images}

Monoscopic restitution systems are designed in order to obtain metric restitution of objects from multiple images acquired by different methods and by different shooting angles and positions.

To obtain metric documentation, these systems can also consider camera internal orientation parameters and objectglass or scanner distortions. These systems are used with multiple images in order to obtain the significant point coordinates of an investigated object by using the images available. From these points it is possible to obtain the dimensions that characterise the object, the metric documentation and a three-dimensional representation, Ortho-photos, photo-plans and three-dimensional photo-realistic models are typical products. The PhotoModeler software is an example of these systems (an application is described below). It works with the following methodology: camera calibration with a specific internal module, acquisition of two or more photographic images from different angles, homologous point identification on different images, 3D model geometry tracking (points, lines, curves, etc.), data processing for 3D model reconstruction, vectorial or photo-realistic 3D model visualization, re-projected on a plane (ortho-photo), extraction of coordinates and shapes, export of various formats for animated rendering or otherwise.

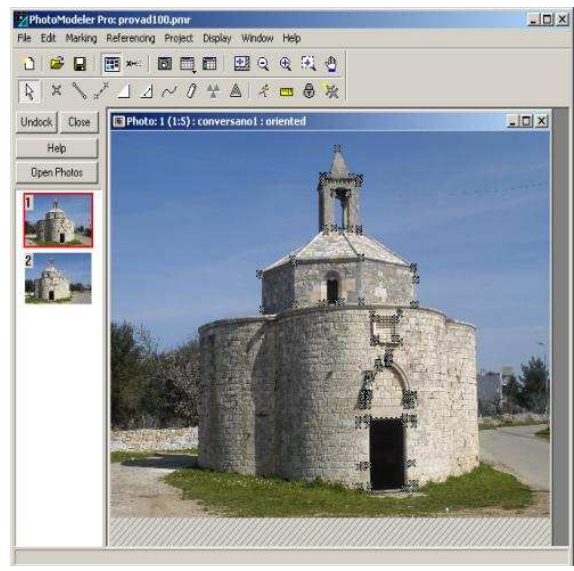

Figure 1: Photomodeler by Eos Sys. 


\subsection{Stereoscopic restitution System}

Stereoscopic photogrammetrical restitution systems have been in use for long time. However the transition from analogical to digital procedures allowed the automation and the simplification of procedures and this is good news. The transition from analogical to digital photogrammetry permits a reduction in costs and difficulties. Final users ask for three-dimensional models of the architectural objects with some metric and semantic information. There is continuous development of easier and cheaper solutions as well as automatic procedures. Stereoscopic restitution Systems are based on:

- $\quad$ stereo pairs acquisition of objects by cameras (note: camera interior-orientation parameters must be known and must be obtained by appropriate calibration);

- digital stereo pairs introduction in the stereo vision system by a special polarization monitor, in order to obtain stereoscopic vision;

- external orientation resolution in two phases (relative and then absolute orientation);

- three-dimensional geometry restitution by the graphic entities plan, through the overlap of pictures and, eventually, through feature discrimination using layers and codes.

The most interesting features are connected with automated processes and final realizable products.

The extraction of point coordinates on an object surface is possible using automatic image correlation and provides a digital model (DSM), usually in a grid format, with a variable level of detail, according to the representation needs and computation time that increases with increasing grid resolution.

If the surface information on the survey object is already available, in this kind of software it is possible to re-project the digital images in order to obtain high accuracy ortho-photos or photo-plans in an easy way.

\section{SURVEY WITH 3D LASER SCANNER}

The spread of Laser scanning is relatively recent and is certainly one of the technologies which will show expansion in the near future. The major expectations in the Cultural Heritage field concern terrestrial equipment. There are several kinds of instruments with different accuracy and load capacity (from $\mathrm{mm}$ to $\mathrm{km}$ ). A measurement session with a laser scanner gives a set of three-dimensional coordinates, usually in a reference system associated with the instrument. These coordinates represent the very high number of points hit by the laser. Therefore the point cloud so generated describes external surface of the scanned object. Laser systems are almost completely automated and they are able to acquire a remarkable number of points per second, sometimes thousands. A laser scan product consists of one or more "point clouds " with high density, thousands or hundreds of thousands of points, which describe object surface and its surrounding environment in great detail (many tools can scan 360 degrees horizontally with high vertical aperture). However, this data is not a stand-alone product like classic graphical representations derived from direct or photogrammetrical surveys, in which the picture is filtered by the operator who selects and codes interesting elements. So software is the key to successful laser scanning but it is often characterized by many unknowns. In fact there are currently some good products but many lack software, especially concerning the architectural heritage field. There are about a dozen main packages currently used, including an Italian product. These are typically stand-alone software products but some are integrated into CAD software as add-on packages. These products integrate some tasks that are not normally included in CAD or three-dimensional data (DTM) management software. Some objects such as sculptures could require a full-3D wire-frame system (with its computational power requirements) rather than a regular planimetric approach (known as a 2.5D approach used for terrain modeling). The stage of "mesh" generation is one of the most delicate and results provided by different programs on the same object can be quite different. Furthermore, in the cultural heritage application field, the techniques of "photorealistic texture mapping" have great importance. Texturing on the 3D model surface, reconstructed from metric laser scanning, is also helpful in the study and interpretation of an object, because we must remember that in many cases the photographic image contains very valuable information about conservation status such as aspect, colors, water traces, etc. The use of high-accuracy orthophotos (true orthophoto), generated from many different pictures, is another interesting development. This allows both full photo coverage of an object and knowledge of shadows or hidden parts, with the possibility of deleting any elements disturbing the final product. We can make some observations about the use of laser scanning in common architectural heritage survey contexts:

- $\quad$ scanner choice depends on the object size, acquisition distance and required precision;

- technology provides high speed automatic acquisition, and does not need a related topographic survey if using a local reference system.

- full object description requires more acquisitions from different stations, to identify any gaps caused by shape (especially for all around objects, e.g. sculptures) and therefore a survey should be carefully planned; the use of a high-reflectance target (flat or spherical) is very common, allowing unification of several scans;

- logistical problems (transportation, instrument weight, power supply, needs to work at night or in low light with triangulation instruments or phase measurement) can be very stringent in certain circumstances. 
Finally, the cost of tools and software packages is still high, and it is hard for a user to buy two or three different types of tool to use them effectively in every possible survey in the cultural heritage field.

\section{EXPERIENCES}

Over the years, some surveys and several experimental tests have been conducted in different localities of Puglia and Basilicata.The first concerns the Church of St. Catherine, situated in Conversano (Bari), which includes many features useful in our studies. The church: has a simple four-sided plan and it is interesting mainly for the presence of large curved surfaces that are more difficult to detect; has a quite limited size; and is a free-standing building, which allows a complete three-dimensional survey. The measurements were made with three laser systems and the results are reported below.

\subsection{Surveys}

Riegl LMS Z210 laser scanner model captures data with a $360^{\circ}$ horizontal angle, $80^{\circ}$ vertical angle, minimum grid scan equal to $2.5 \mathrm{~mm}$ and a minimum 80 mgon angle. Cyrax 2500 Laser Scanner captures data with a $40^{\circ}$ horizontal and vertical angle, but unlike the previous one, with a minimum grid scan equal to $4 \mathrm{~mm}$, and in this case, the tool works with time of flight method. The GS 100 3D laser scanner made by the French company Mensi is a tool scanning up to 100 meters with a $360^{\circ}$ horizontal angle (the same as the Riegl) and a $60^{\circ}$ vertical angle.

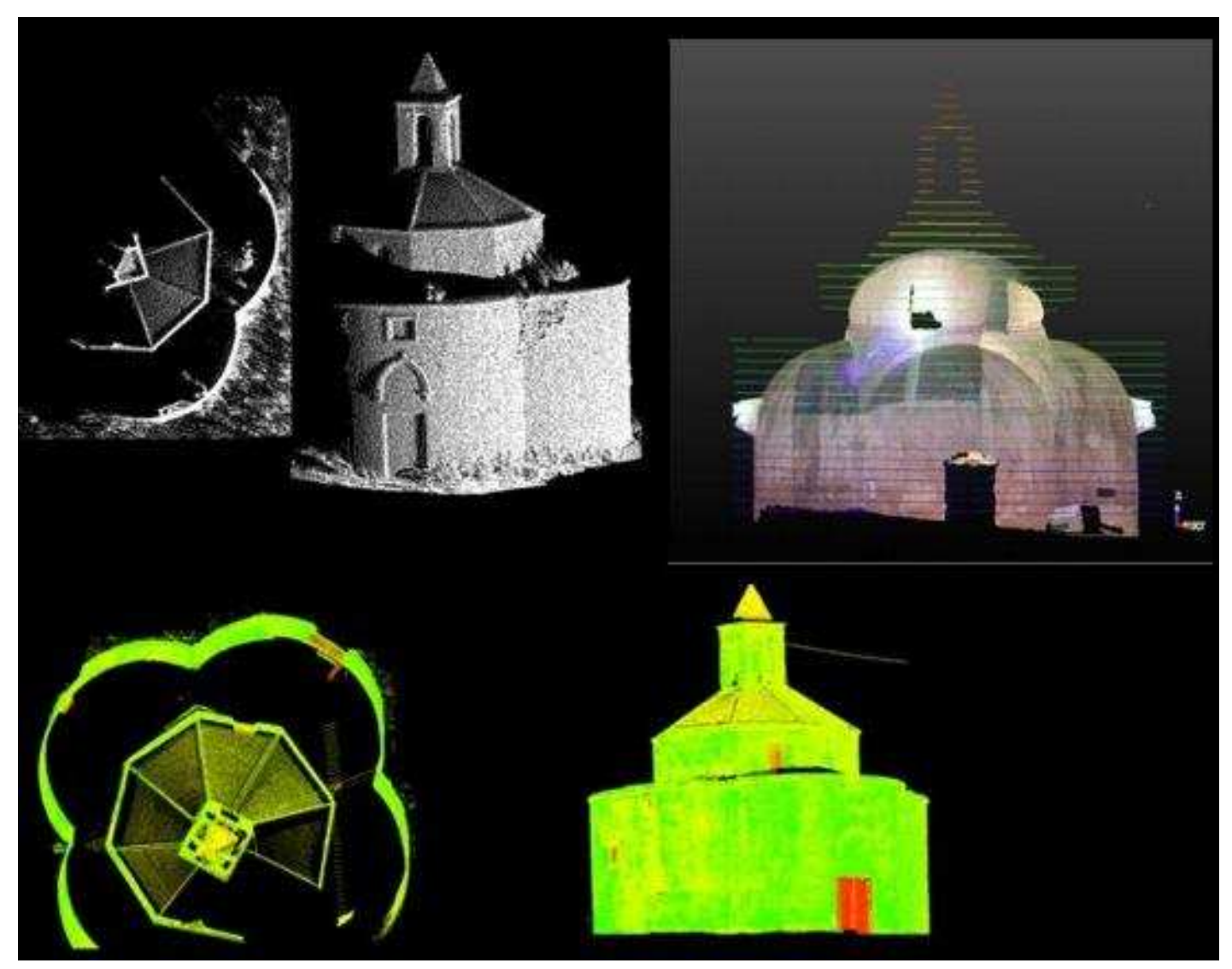

Figure 2: Point Clouds with different instruments

These points, detected in abundant quantities, efficiently and completely describe the surface of our Church, however, the abundance of data generates a certain difficulty in identifying significant points of detected architecture, just as we are accustomed to recognizing in classical architecture representations realized with vectors that conventionally describe object such as edges, slits, overhangs, etc. Further experience and 3D laser scanning instruments allowed the development and refinement of the most effective methodologies for using this survey technique. The following pictures were shot with a Leica HDS Laser in the UNESCO World Heritage Site of "Sassi" in Matera. 


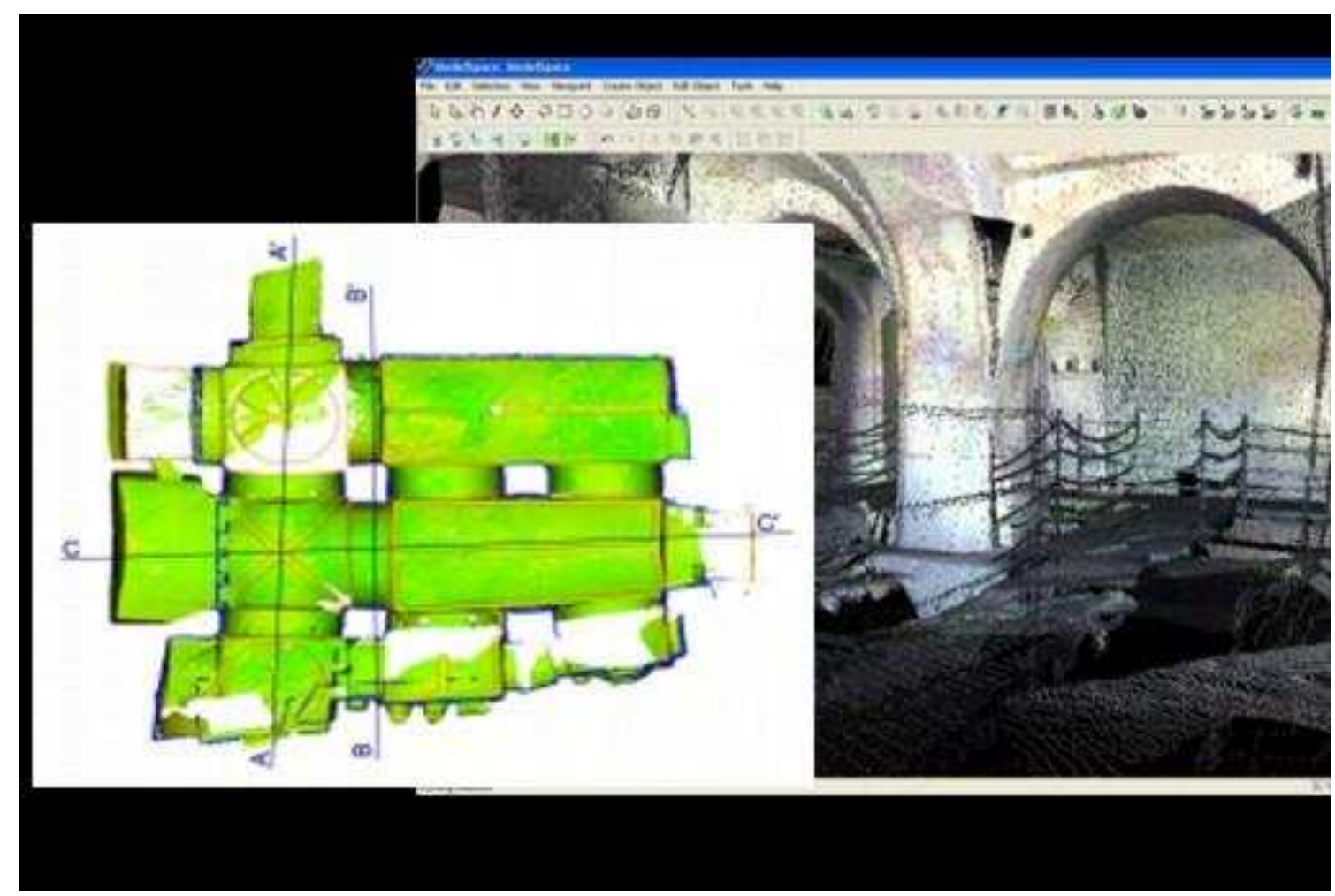

Figure 3 "Convicino S. Antonio "- Matera

The next image was obtained using Topcon's new generation Total Station. Its motorized system allows acquisition of a large number of coordinates. This tool, less expensive than laser scanners, in some cases can usefully replace 3D laser scanning, and obtain an already reduced point cloud integrated with the traditional topographic survey.

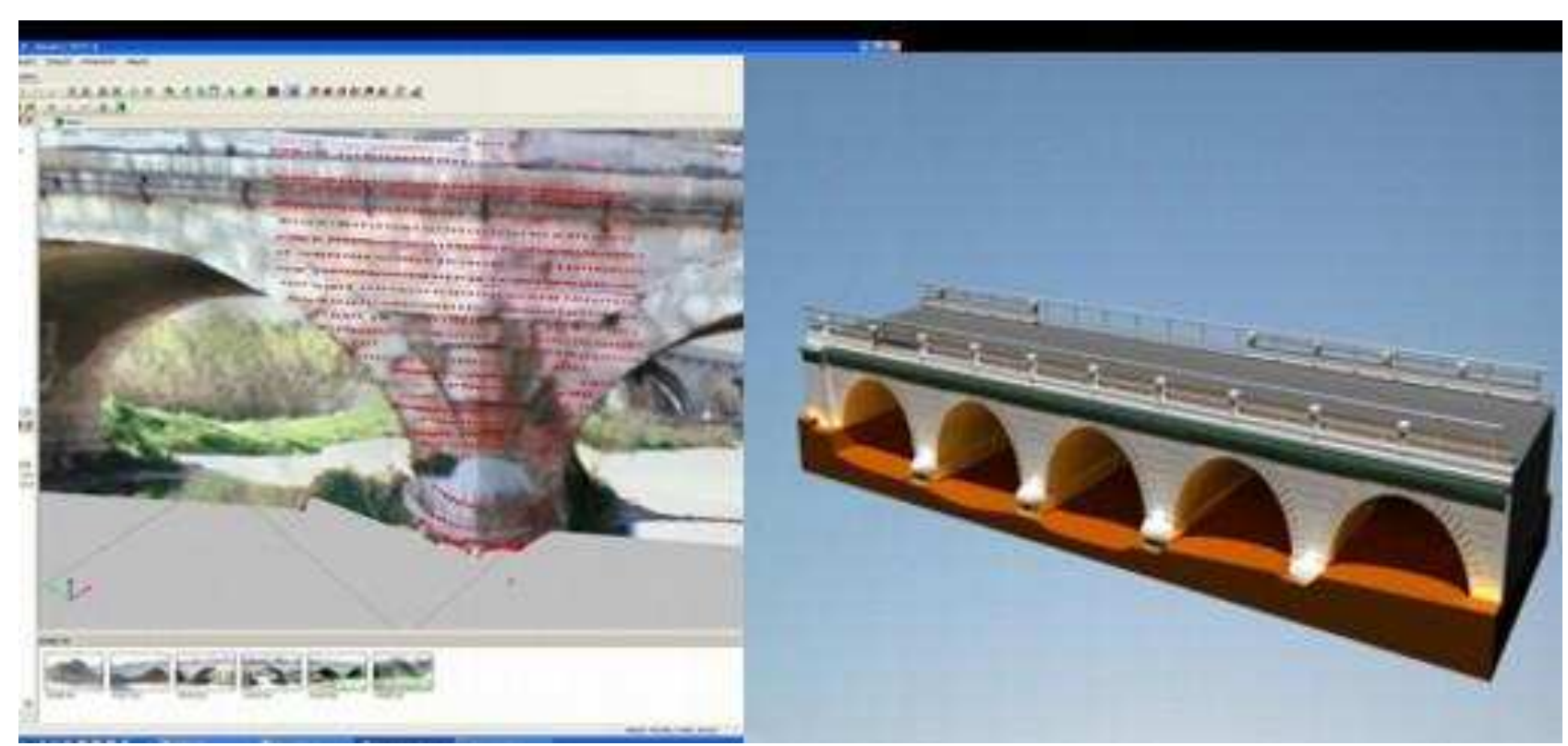

Figure 4: Stone bridge "San Giorgio" - Bari-Topcon IS (Imaging Station) and 3D model 
The next figure shows the results of the survey of the cave church of St. Vigilia in a GIS environment and then extracting sections, elevations and floor plans.

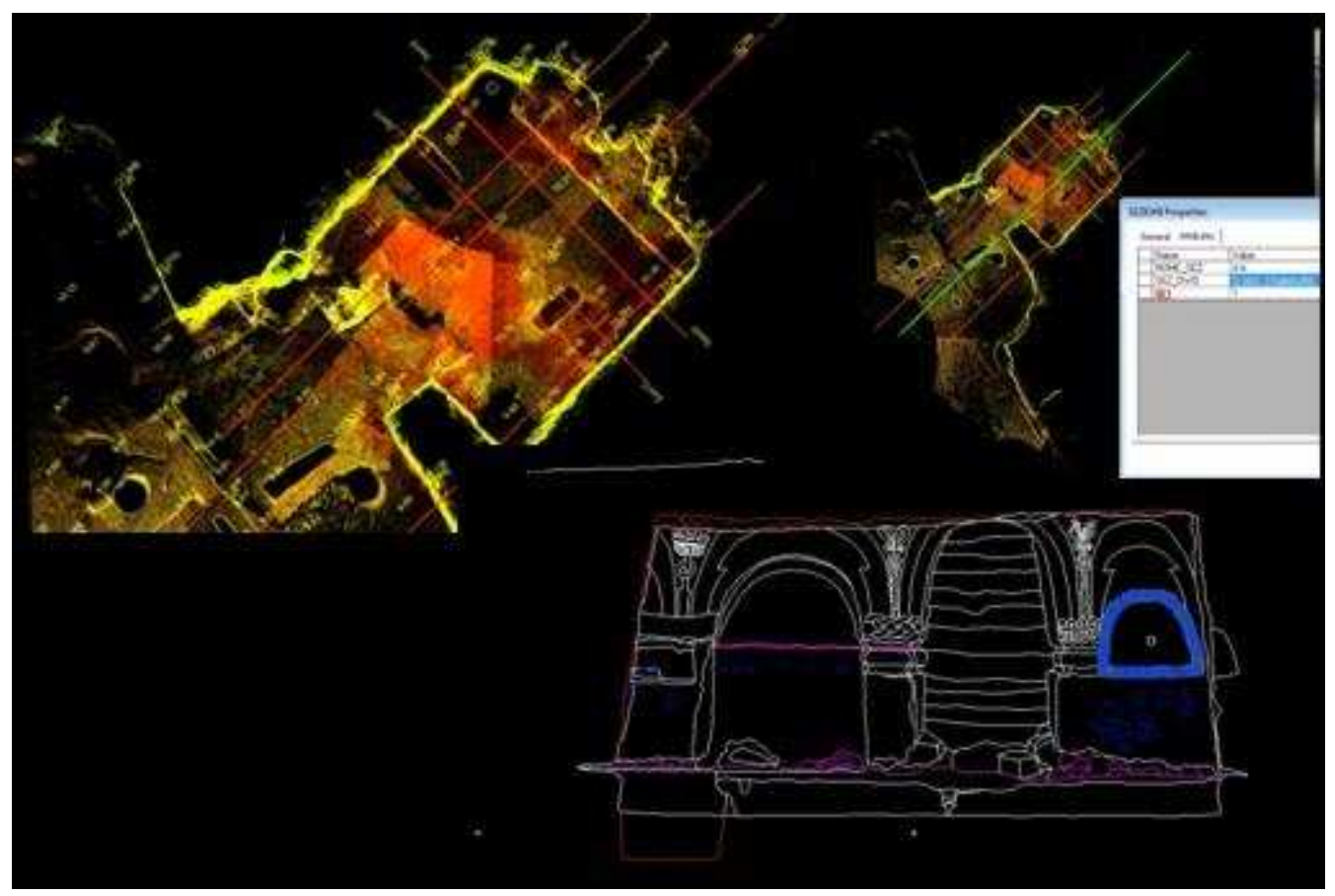

Figure 5: Point cloud of S.Vigilia cave church, Fasano (BA) in Gis environment and section extraction

\section{CONCLUSIONS}

Experience since 1980 in the field of architectural photogrammetry, lead us to make some comments about the use of photogrammetical systems or laser scanners. Comprehension and execution of the photogrammetrical approach was, and still is, quite difficult for a new user. Photographic equipment for image acquisition requires a geometrical study and radiometric calibration which are hard to automate. Previous considerations cannot be overlooked, although current methods allow many more placement patterns and refined automation of camera exposure. Laser scanning computer processes, with high-speed data acquisition, have made the terrestrial photogrammetrical technique falsely obsolete and "unfashionable". The great demand for laser acquisition of historical buildings, monuments and objects for documentary purposes, underlines the previous observations. But as usually happens, increasing demand is not followed by training the purchaser in the requirements and goals for a correct survey. Despite the great potential of these tools, they are often only used to produce plans, elevations and sections (plotted to scale!). We believe that photogrammetry in the near future may still give a significant contribution especially to architects (and archaeologists), who require, as specialists, to be able to interpret the object of their interest and not just measure it.

\section{REFERENCES}

[1] Dequal, S., et A, "The solid image: an easy and complete way to describe $3 \mathrm{~d}$ objects. International Archives of the Photogrammetry, Remote Sensing and Spatial Information Sciences, XX ISPRS, Istanbul. 2004

[2] Galetto, R, Camere Digitali per le riprese aeree e terrestri, Bollettino SIFET n. 42004.

[3] Caprioli, M., Scognamiglio A. "Low coast methodology for 3D modelling and metric description in architectural heritage", IAPRS Vol XXXVIII 2009 